\title{
Valoración de indicadores para la CT-03 Análisis y resolución de problemas
}

Vidal-Meló, Anna a , Boigues Planes, Francisco J. ${ }^{\mathrm{b}}$ y Pastor Gimeno, José I.

Universitat Politècnica de València, Departamento de Matemática Aplicada, Grup d'Innovació Educativa i Recerca en Matèries Cientifiques (GIERMAC). ${ }^{a}$ avidal@mat.upv.es, ${ }^{b}$ fraboipl@mat.upv.es y ${ }^{c}$ jpastogi@mat.upv.es

\section{\$EWWFW}

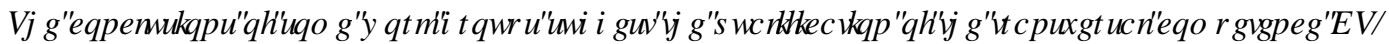

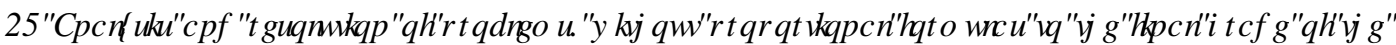

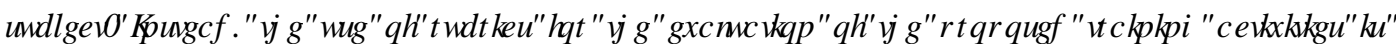

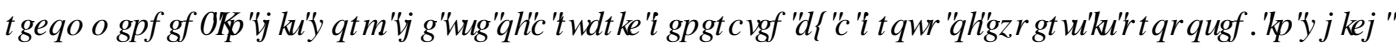

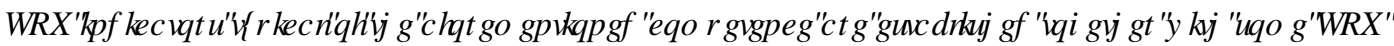

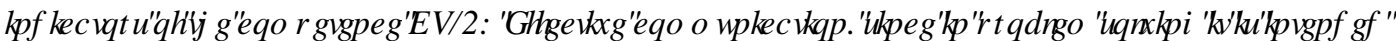

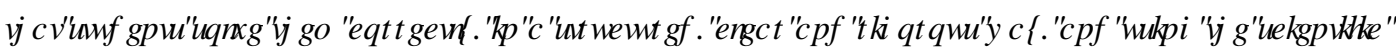

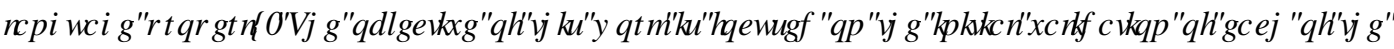

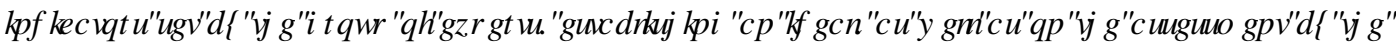

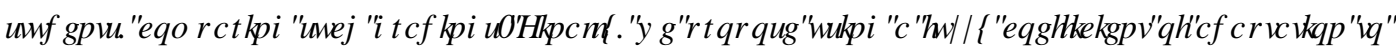

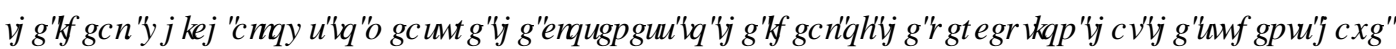

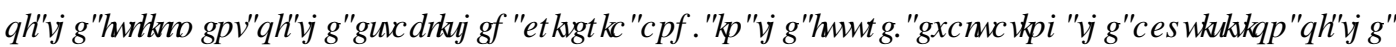
DRLP HQURQHGFRP SHAQFHIQWKHWWGHWD

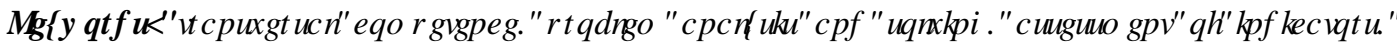
WWGHWSHFH WRQLIX]]। $P$ P DWXUH

प

\section{HXP HQ}

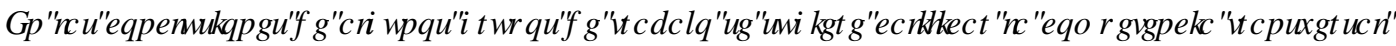

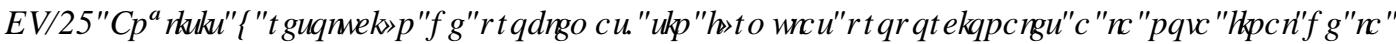

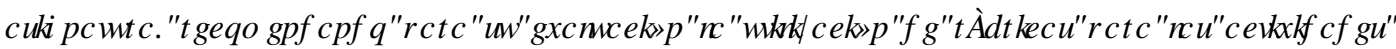

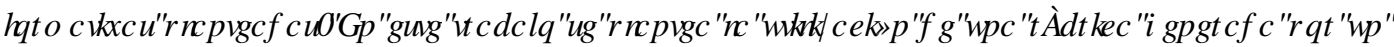

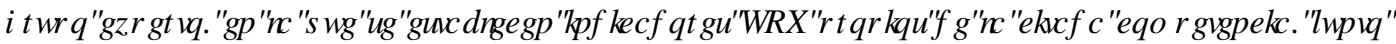

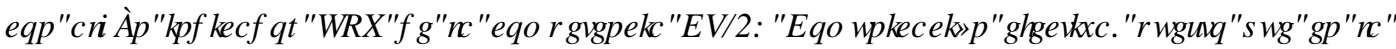

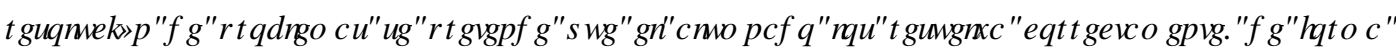

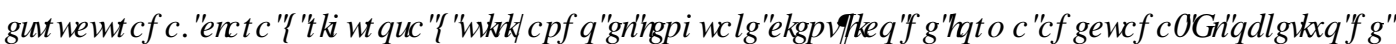

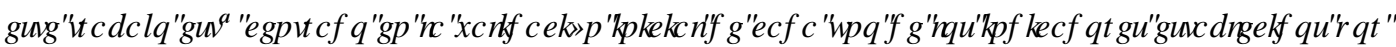

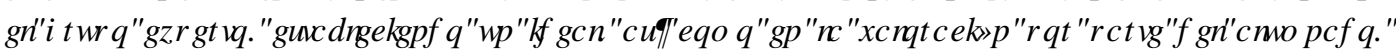

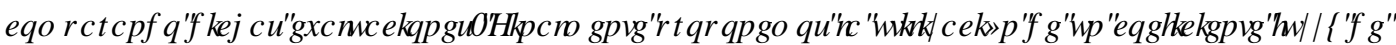

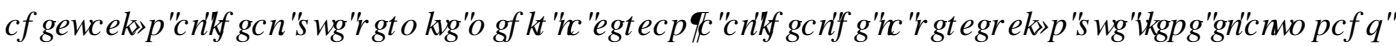

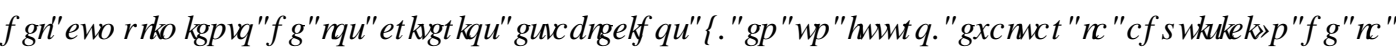
$P$ HQFIRQDODFRP SHMQFIDHQHODOX QDORD

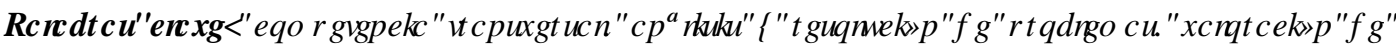

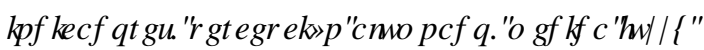




\section{Introducción}

Las competencias transversales son aquellas habilidades relacionadas con el desarrollo personal, que no dependen de un ámbito temático o disciplinario específico sino que aparecen en todos los dominios de la actuación profesional y académica (González y Wagenaar, 2003). El Proyecto Competencias transversales de la UPV (UPV, 2015) considera un total de 13 competencias transversales, CT. El presente trabajo se centra en una de ellas, la CT-03 Análisis y resolución de problemas, referida a la capacidad de analizar y resolver problemas de forma efectiva, identificando y definiendo los elementos significativos, con el objetivo de que el alumnado sea capaz de aplicar procedimientos estructurados para resolver problemas, promoviendo así su capacidad de aprender, comprender y aplicar conocimientos de forma autónoma.

Existe diversidad de trabajos de estudio de las CT, entre ellos trabajos de la Universitat Politècnica de València, en los que se analizan en particular la competencia CT-03 Análisis y resolución de problemas (Álvarez et al., 2016; Cervelló y Marqués, 2016; Pérez-Sánchez, Pérez-Sánchez y López 2017; Calafat et al., 2019; Cortes et al., 2019). En cuanto a las actividades formativas utilizadas para su evaluación, destacan las mismas pruebas o exámenes parciales de la asignatura, la evaluación a través de las prácticas de la asignatura y en otros casos una serie de ejercicios realizados en clase que son útiles para valorar la competencia y que sirven de preparación para evaluarla finalmente en los parciales de la asignatura (Álvarez et al., 2016). Para evaluar la CT en el mismo examen, algunas asignaturas incorporan ciertas preguntas a algunos apartados de los problemas del examen de manera que las pruebas de evaluación de la asignatura recogen algún apartado específico para la evaluación de la CT. En cuanto a los instrumentos de evaluación, en el caso de las rúbricas, en algunas se consideran los mismos indicadores de las rúbricas del Site "Competencias Transversales UPV". Otras establecen sus propios indicadores o resultados de aprendizaje, adaptados a su asignatura:

- Aplica correctamente las técnicas de resolución; interpreta y analiza los resultados; expone ordenadamente las ideas; sabe expresarse por escrito con corrección (Cortes et al., 2019);

- Define el problema e identifica los datos correctamente; emplea un método aprendido para la resolución del problema propuesto (Cervelló y Marqués, 2016);

- Determina si algún dato es innecesario, redundante o contradictorio; realiza un esquema o gráfico que ayude a la comprensión del problema; realiza una verificación rápida del resultado para detectar posibles errores durante el desarrollo; determina si un problema puede resolverse de varios modos y valora cada una de las alternativas. (Álvarez et al., 2016)

A pesar de todo, mayoritariamente se observa que la calificación de la adquisición de la CT se lleva a cabo a partir de la nota obtenida en el examen, de manera proporcional a la calificación. En las conclusiones de Álvarez et al. (2016) se sugiere calificar las competencias sin fórmulas proporcionales a la nota final de la asignatura. Es pues recomendable la utilización de rúbricas para las diferentes actividades formativas planificadas, y así lo tuvimos en cuenta en nuestro Proyecto de Innovación y Mejora Educativa (PIME) 1920/190 Competencias transversales en asignaturas físico-matemáticas consideradas punto de control: desarrollo de actividades, recoger evidencias y evaluar sin morir en el intento, en el que participamos un grupo de 6 profesores del Departamento de Matemática Aplicada y de Física Aplicada de la Escola Politècnica Superior de Gandia (EPSG). Entre los objetivos específicos del proyecto destacamos el estudio detallado sobre los criterios de evaluación aplicados al estudiante en nuestras asignaturas acerca de la adquisición de las CT correspondientes, la realización de encuestas competenciales a nuestro alumnado sobre la percepción sobre su nivel de adquisición y estudiar las posibles desviaciones respecto de la evaluación del profesor o profesora.

Para conformar nuestros indicadores al menos se deben tener en cuenta las 4 fases de la resolución de problemas (Polya, 2014), aplicables al resto de disciplinas técnicas: Entender y explorar el problema, 
encontrar una estrategia y utilizarla para resolver el problema y finalmente reflexionar sobre la solución. Estas fases vienen reflejadas en los diferentes indicadores de las rúbricas establecidas por la UPV para la evaluación de la CT-03 y en los indicadores de los trabajos estudiados. Sin embargo se precisa añadir alguno más relacionado sobre todo con otra de las competencias, la CT-08 Comunicación efectiva. En las Figuras 1 y 2 se muestran formas diferentes de resolver dos problemas, uno consistente en verificar si es cierta o no una información de internet que indica que en el túnel de Guadarrama, con velocidad limitada de $100 \mathrm{~km} / \mathrm{h}$, cualquier vehículo que lo haga en menos de 2 minutos y 5 segundos será multado y el otro relacionado con los Objetivos de Desarrollo Sostenible (ODS) en el que se desea encontrar la lata cilíndrica más sostenible en cuanto a la menos superficie empleada. En la Figura 1 no se ve estructura alguna, limitándose a cálculos correctos o no, sin finalizar con la correspondiente conclusión o con resultados no comprobados. En la Figura 2 se observa un proceso más completo con una buena comunicación escrita, además de aportar dos formas de resolución en el primer problema y una estructura detallada en el segundo.

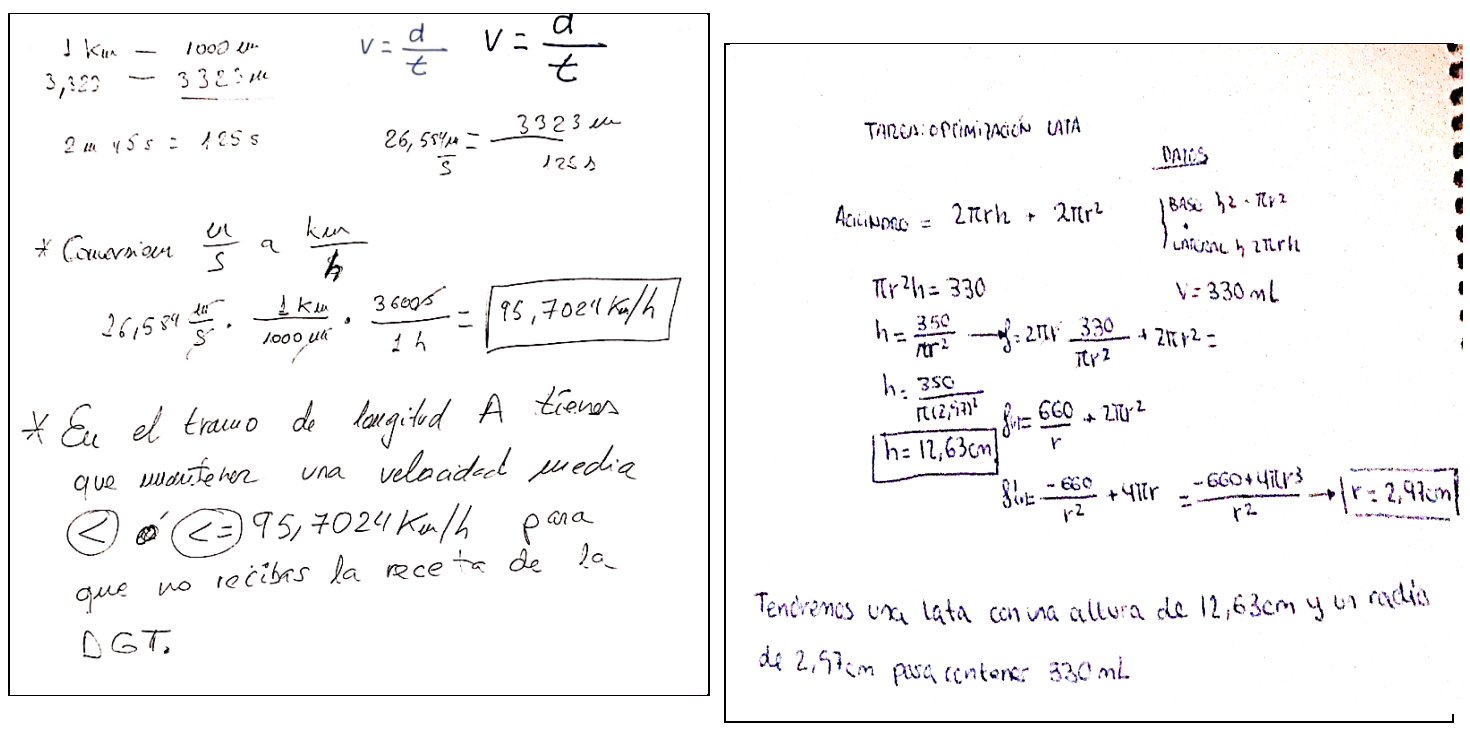

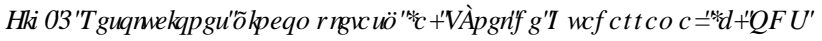

(1)

Indicadores como "expone ordenadamente las ideas y sabe expresarse por escrito con corrección" (Cortes et al. 2019), también deben tenerse en cuenta, indicadores que aparecen en las rúbricas de la CT-08 Comunicación efectiva, como se ve en las Figuras 3 y 4. La competencia CT-08 significa tener desarrollada la capacidad de transmitir conocimientos y expresar ideas y argumentos de manera clara, rigurosa y convincente, tanto de forma oral como escrita, utilizando los recursos apropiados adecuadamente y adaptándose a las circunstancias y al tipo de público. Observamos por tanto que en la resolución de problemas es esencial saber explicar la resolución, no limitarse únicamente a una acción asociativa y rutinaria. El objetivo es que el alumnado resuelva los problemas de forma estructurada, clara y rigurosa, utilizando el lenguaje literario-matemático de forma adecuada. La comunicación efectiva del alumnado constituye una evidencia del aprendizaje y un elemento a considerar también como refuerzo al propio aprendizaje, ya que el esfuerzo para explicar lo que sabe conlleva un proceso de interiorización que facilita un aprendizaje más robusto y significativo (Skemp, 1978). 
Se nos presentar el caso de suno de los 3

tiveles de Guadarrama.

Este va de Madred (punto A) a Castille yleón (punto B). La distancia entre ambos sin puntos es de $3,323 \mathrm{~km}$ y la veeocidad máxima permitide de $100 \mathrm{~km} / \mathrm{h}$.

$$
\text { (A) } \underbrace{\ldots . . .1(3)}_{3,323 \mathrm{~km}}
$$

Vamos a suponer que nuestro velículo va de verocidad media a exos $100 \mathrm{~km} / \mathrm{h}$, tenemos que ba velocidand meclia es iguale a le distan cia recorride partido el tiempo que se he tarda do, por tanto:

$v_{m}=\frac{\Delta x}{\Delta t} \rightarrow V_{m}=100 \frac{\mathrm{km}}{\mathrm{h}} \cdot \frac{1 \mathrm{~h}}{3600 \mathrm{~s}}=\frac{1}{36} \frac{\mathrm{km}}{\mathrm{s}}$

$\frac{1}{36}=\frac{3,323}{\Delta t} \rightarrow \Delta t=3,323 \cdot 36=1191628 \mathrm{~s}$

Es decir, si fuesemos a velocidad media de $100 \mathrm{~km} / \mathrm{h}$ durante $3,323 \mathrm{~km}$, tar dariamos 1 minuto y 59 segundos en craticurlo.

Li suponemos el tiempo por el cual se empieza a mullar ( 2 min y 5 seg. ) ba velocidend media má xima permilida el de:

$V_{m}=\frac{3,323}{\frac{5}{144}}=9517024 \mathrm{~km} / \mathrm{h}$

Por tante, ob servamos que la velocidad media para recoxver el tûnele en 2 minys 5 es de $95.7024 \mathrm{~km} / \mathrm{h} ; \mathrm{y}$ que en realidad, si frexemos min Ey conclurión, la información es crovea.

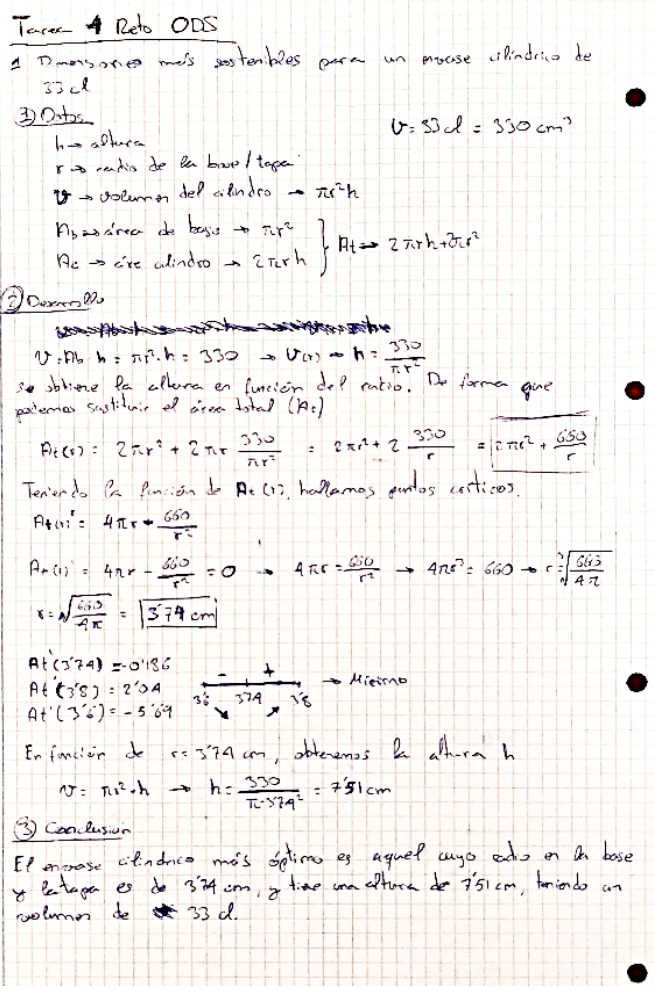

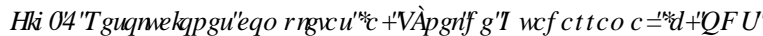

RÚBRICA UPY CT-OB.COMUNICACIÓN EFECTIVA

DIMENSIÓN COMUNICACIÓN ORAL - Nivel de dominio I

Resultodo de oprendizaje: expresar ideas de forma estructurada e inteligble en presentaciones orales de duración breve o en intervenciones puntudes.

\begin{tabular}{|c|c|c|c|c|c|}
\hline \multirow{2}{*}{ INDICADORES } & \multicolumn{4}{|c|}{ DESCRIPTORES } & \multirow{2}{*}{$\begin{array}{l}\text { EJEMPIOS DE } \\
\text { POSIBLS EVIDENCIAS }\end{array}$} \\
\hline & D. No alcanzodo & C. En desarrollo & B. Bien /adecuado & A. Excelente/ejemplar & \\
\hline $\begin{array}{l}\text { Muestra una } \\
\text { disposición persanal } \\
\text { fovoroble hacia la } \\
\text { comunicoción }\end{array}$ & $\begin{array}{l}\text { No interverene ircluso } \\
\text { cuando es interpelodo o } \\
\text { lo hoce faltando a las } \\
\text { normas bósicas de } \\
\text { educociśn y convivencia }\end{array}$ & $\begin{array}{l}\text { Interviere solo cuondo es } \\
\text { interpelado y/o } \\
\text { manifiesta actitudes poco } \\
\text { apropiadas }\end{array}$ & $\begin{array}{l}\text { Interviene de manera } \\
\text { voluntaria y evidencia } \\
\text { indicios de escucha } \\
\text { acfiva }\end{array}$ & $\begin{array}{l}\text { Participa habitualmente } \\
\text { con iniciativa y } \\
\text { oporturidad }\end{array}$ & $\begin{array}{l}\text { No monifesta octhides como } \\
\text { sobstitia desgona desinterts. } \\
\text { perdida de alonción.. }\end{array}$ \\
\hline $\begin{array}{l}\text { Tronsmite inicrmacion } \\
\text { relevonte y sabe } \\
\text { responder las } \\
\text { preguntas que se ie } \\
\text { formubn }\end{array}$ & $\begin{array}{l}\text { Se expreso de manero } \\
\text { pobre y/o contusa y ro } \\
\text { sabe responder los } \\
\text { creguntas que se le } \\
\text { formulan }\end{array}$ & $\begin{array}{l}\text { Presenla solo algunas } \\
\text { ideos o de monero } \\
\text { insuliciente y, pese o } \\
\text { contestorias, no llegas } \\
\text { responder los preguntas } \\
\text { que se le formulan }\end{array}$ & $\begin{array}{l}\text { Espore los ideas mós } \\
\text { mportantes y sabe } \\
\text { resconder comectamente } \\
\text { a las preguntas que se le } \\
\text { tormulan }\end{array}$ & $\begin{array}{l}\text { Presenta una perspecfiva } \\
\text { gobad y fundamento } \\
\text { adecuodamente los ideos } \\
\text { que expone, respondendo } \\
\text { con ocierto los preguntos } \\
\text { que se le formulan }\end{array}$ & 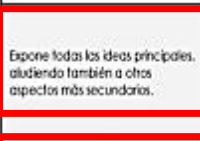 \\
\hline $\begin{array}{l}\text { Los exposiciones están } \\
\text { esiructurodos de modd } \\
\text { caherente }\end{array}$ & $\begin{array}{l}\text { La exposición evidencia } \\
\text { folta de orden y/o } \\
\text { jeraquia en la } \\
\text { presentosión de sus ideas }\end{array}$ & $\begin{array}{l}\text { Lo estuctura de la } \\
\text { presentoción no tocilia su } \\
\text { comprersión }\end{array}$ & $\begin{array}{l}\text { Los exposiciones estón } \\
\text { estruchurodos de un } \\
\text { modo coherente, que } \\
\text { focita su comprersión }\end{array}$ & $\begin{array}{l}\text { Lo exposición resulta } \\
\text { organizada, completay } \\
\text { eficoz }\end{array}$ & 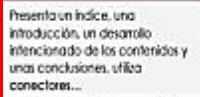 \\
\hline
\end{tabular}

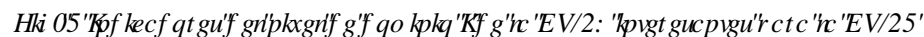

DIMENSIÓN COMUNICACIÓN ORAL - Nivel de dominio II

Resultado de oprendizaje: transmitir convicción y seguridad, ilustrar el discurso para focíltar su comprensión y odaptarlo a las condiciones formales exigidas en presentaciones orales de duración media (10-15 minutos aproximadamente).

\begin{tabular}{|c|c|c|c|c|}
\hline \multirow{2}{*}{ INDICADORES } & \multicolumn{4}{|c|}{ DESCRIPTORES } \\
\hline & D. No alconzado & C. En desarrolo & B. Blen /adecuado & A. Excelente/ejemplar \\
\hline $\begin{array}{l}\text { Respeta las normas } \\
\text { aingüisticos } \\
\text { (corrección/ y se } \\
\text { expresa de manera } \\
\text { correcta (claridad) }\end{array}$ & 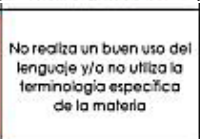 & 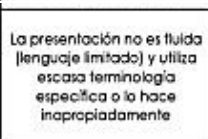 & $\begin{array}{l}\text { Respeta los normas } \\
\text { ingu'stcas y se expresa } \\
\text { do manera correcta. } \\
\text { utlizando de modo } \\
\text { pertinente la } \\
\text { terminologia espeofica }\end{array}$ & $\begin{array}{l}\text { La exposición se adopta a } \\
\text { la oudiencia, hociondo uso } \\
\text { de un lengudo y do una } \\
\text { ferminologio especilicos. } \\
\text { apropiodos, precisss y ricos }\end{array}$ \\
\hline
\end{tabular}

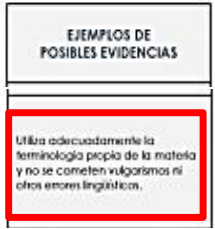

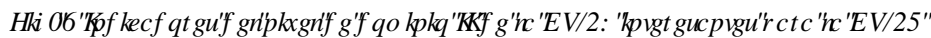

(cc) EY-NC-ND 2021, Universitat Politècnica de València

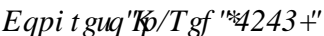


Así pues en nuestro estudio pensamos que era necesario combinar rúbricas e incorporar algunos indicadores, de gran interés en la resolución de problemas, correspondientes a la CT-08: la importancia de fundamentar las ideas, responder a las preguntas planteadas (es habitual que al final no contesten a las preguntas formuladas, limitándose a la realización de cálculos), la resolución debe estar estructurada y organizada y finalmente es importante la utilización de la terminología propia de la materia y del lenguaje matemático.

Uno de los resultados parciales de nuestro PIME ha sido el consenso sobre 8 indicadores o criterios para poder evaluar la CT-03, indicadores presentados al alumnado de una asignatura de matemáticas durante el curso 2019-2020 a través de la estructura de la Tabla 1.

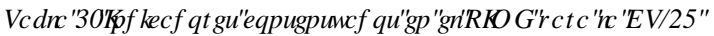

\begin{tabular}{|l|}
\hline \multicolumn{1}{|c|}{ INTRODUCCIÓN O PLANTEAMIENTO } \\
\hline Criterios \\
\hline 1. Identifica claramente el objeto del problema, modelizando matemáticamente el problema a resolver \\
\hline 2. Describe el método o estrategia de resolu ción según lo estudiado \\
\hline Criterios \\
\hline 3. Justifica los cálculos en cada paso de la resolución \\
\hline 4. Utiliza correctamente la notación y termin ologia adecuada \\
\hline 6. Es eficiente en alcanzar la solución la solución correcta cro \\
\hline 7. Describe, argumenta y comenta la solución obtenida \\
\hline 8. Responde claramente a la cuestión planteada \\
\hline CONCLUSIÓN \\
\hline Criterios \\
\hline
\end{tabular}

Durante el curso se propusieron varias Tareas (Túnel de Guadarrama, ODS,...) en las que debían resolver un problema siguiendo una estructura similar a la de un relato, con las siguientes partes:

- Un planteamiento donde se expone el análisis de los diferentes elementos del enunciado del problema: describir los datos, definir las variables e incógnitas, interrelaciones entre ellas, y reconocer cuál es la pregunta que hay que responder.

- La parte central o del desarrollo: describir y ejecutar el método de resolución adecuado, sintetizando la información disponible, proponiendo y utilizando el método más adecuado para resolver el problema y justificando los cálculos en cada paso del proceso de resolución.

- Finalmente la conclusión o desenlace: descripción y argumentación de las soluciones obtenidas, respondiendo claramente a la pregunta del enunciado.

La Figura 2 (b) muestra la correcta utilización de este modelo de resolución en uno de las Tareas.

Es fácil observar que los indicadores o criterios reflejados en la Tabla 1 están relacionados con las dos competencias, CT-03 y CT-08. En particular en los criterios 2, 4, 7 y 8 es importante una comunicación escrita eficaz. De esta forma en la resolución de problemas se evalúa no solo el resultado, sino también el proceso primando que la transmisión del mensaje por parte del alumnado se haga de modo claro y entendible, sin generar confusión, dudas o interpretaciones erróneas (Boigues, Estruch y Vidal, 2019). 
En este trabajo se presentan las valoraciones de los criterios fijados para la competencia transversal CT-03 Análisis y resolución de problemas, por parte de un grupo experto y por parte de un grupo de estudiantes en una asignatura de Matemáticas. Después de especificar los objetivos de este trabajo, en la descripción del desarrollo de la innovación se muestran las valoraciones obtenidas por el grupo experto y por el alumnado. Como resultados primero se analizan las valoraciones del grupo experto, estableciéndose la valoración ideal, y las del alumnado para pasar a comparar dichas valoraciones. En segundo lugar implementamos técnicas fuzzy para analizar la adecuación entre las respuestas sobre la percepción de los estudiantes respecto al cumplimiento de los indicadores y del ideal fijado por el grupo experto. Este método también permite obtener el grado de adquisición de la mencionada competencia en el alumnado.

\section{Objetivos}

En este trabajo nos planteamos varios objetivos:

- Valorar los indicadores o criterios establecidos para la evaluación de la CT-03, tanto por un grupo experto como por el alumnado y analizar y comparar dichas valoraciones.

- Medir la percepción del alumnado respecto a la adquisición de la competencia CT-03 a través de un grado de pertenencia al ideal creado por el grupo experto, resultado de la utilización de un coeficiente fuzzy de adecuación al ideal.

\section{Desarrollo de la innovación}

La experiencia tiene lugar en el curso 2019-2020 con estudiantes del Grado en Tecnologías Interactivas, GTI, grado que se imparte en el Campus de Gandia de la Universitat Politècnica de València, más específicamente en la asignatura "Cálculo. Ecuaciones Diferenciales", con 22 estudiantes matriculados. A pesar de estar emplazada en el semestre $3 \mathrm{~A}$, realmente se trata de una asignatura de matemáticas básicas, de 4.5 ECTS, y en la que mayoritariamente se resuelven problemas de una colección de boletines. Además, participaron 9 profesores de los departamentos de Matemática y Física Aplicada, 6 de ellos participantes en el PIME 19-20/190.

\subsection{Toma de datos}

Para la recolección de datos relativos a las valoraciones, se diseñó un cuestionario en el que se debía valorar entre 0 y 10 los indicadores o criterios consensuados por los participantes del PIME:

Criterio 1: Identificar claramente lo que el problema pide que se resuelva y las matemáticas necesarias para su resolución (es como una introducción antes de la resolución enlazando con la teoría)

Criterio 2: Describir el método que hay que utilizar para resolver el problema según lo estudiado (como los pasos generales a seguir)

Criterio 3: Justificar los cálculos en cada paso de la resolución (pasos intermedios o subpasos)

Criterio 4: Utilizar correctamente la notación y terminología adecuada

Criterio 5: Alcanzar la solución correcta (a veces por fallos tontos puede no llegar)

Criterio 6: Ser eficiente en alcanzar la solución (si hay varias alternativas elegir la mejor, la más cómoda, la más rápida...)

Criterio 7: Responder claramente a las preguntas planteadas (en ocasiones se termina en un cálculo pero no se contesta a la pregunta) 
Criterio 8: Describir, argumentar, comentar la solución obtenida y, si es el caso, comprobar si la solución tiene sentido

Dicho cuestionario se les pasó a dos muestras distintas:

- Grupo experto: formado por 9 profesores del Departamento de Matemática Aplicada y de Física Aplicada, tres de ellos fuera del PIME 19-20/190. Se les pidió que valoraran el grado de importancia que le dan a cada uno de los criterios establecidos, entendiendo que dicho valor posteriormente se trataría como un mínimo exigido para la adquisición de la CT.

- Alumnado: 14 estudiantes de la citada asignatura. Estos realizaron dos valoraciones, una como posible grupo experto y otra con la percepción que tenían sobre su grado de cumplimiento de cada uno de los criterios, también entre 0 y 10.

\subsection{Valoración de los criterios por parte del grupo experto y por parte del alumnado}

Inicialmente se consideran las valoraciones en cada uno de los 8 criterios establecidos para la CT-03, por parte del grupo experto, formado por 9 docentes, valoraciones que vienen reflejadas en la Figura 5. Análogamente, la Figura 6 muestra las valoraciones de 14 estudiantes en cada uno de los criterios.
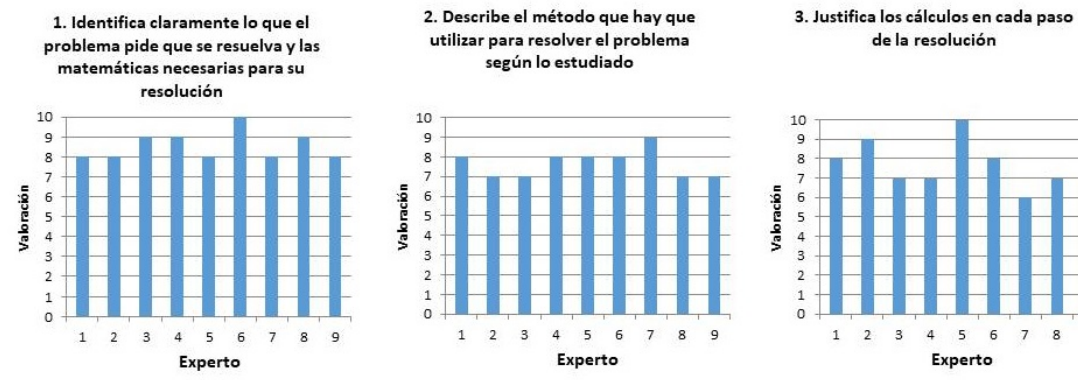

de la resolución

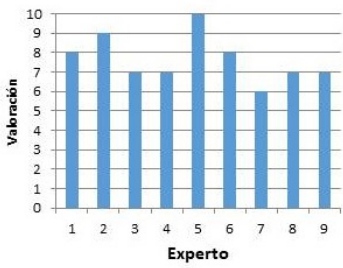

6. Es eficiente en alcanzar la solución
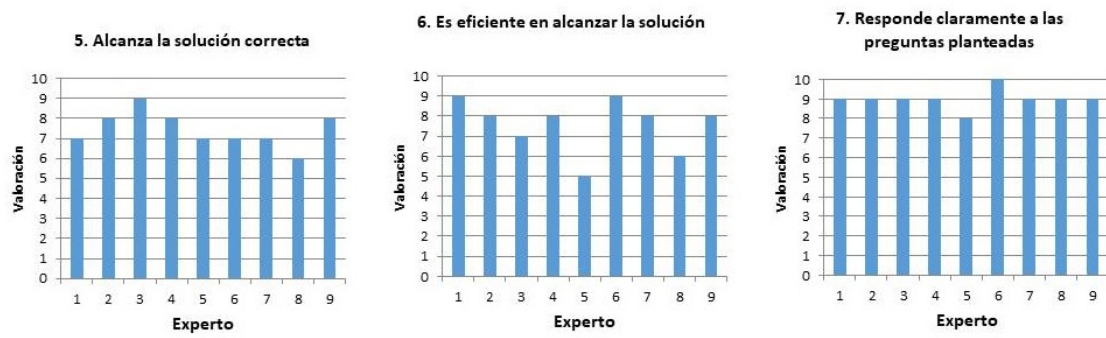

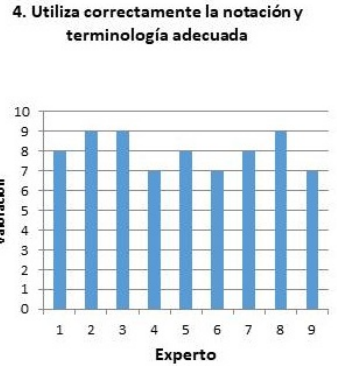

Experto

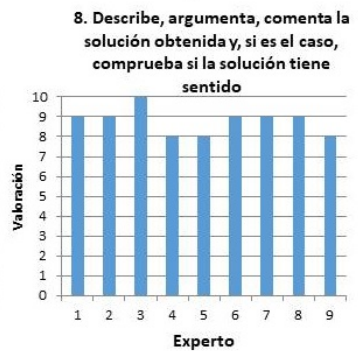

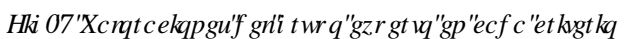

(c)) BY-NC-ND 2021, Universitat Politècnica de València

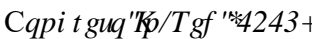



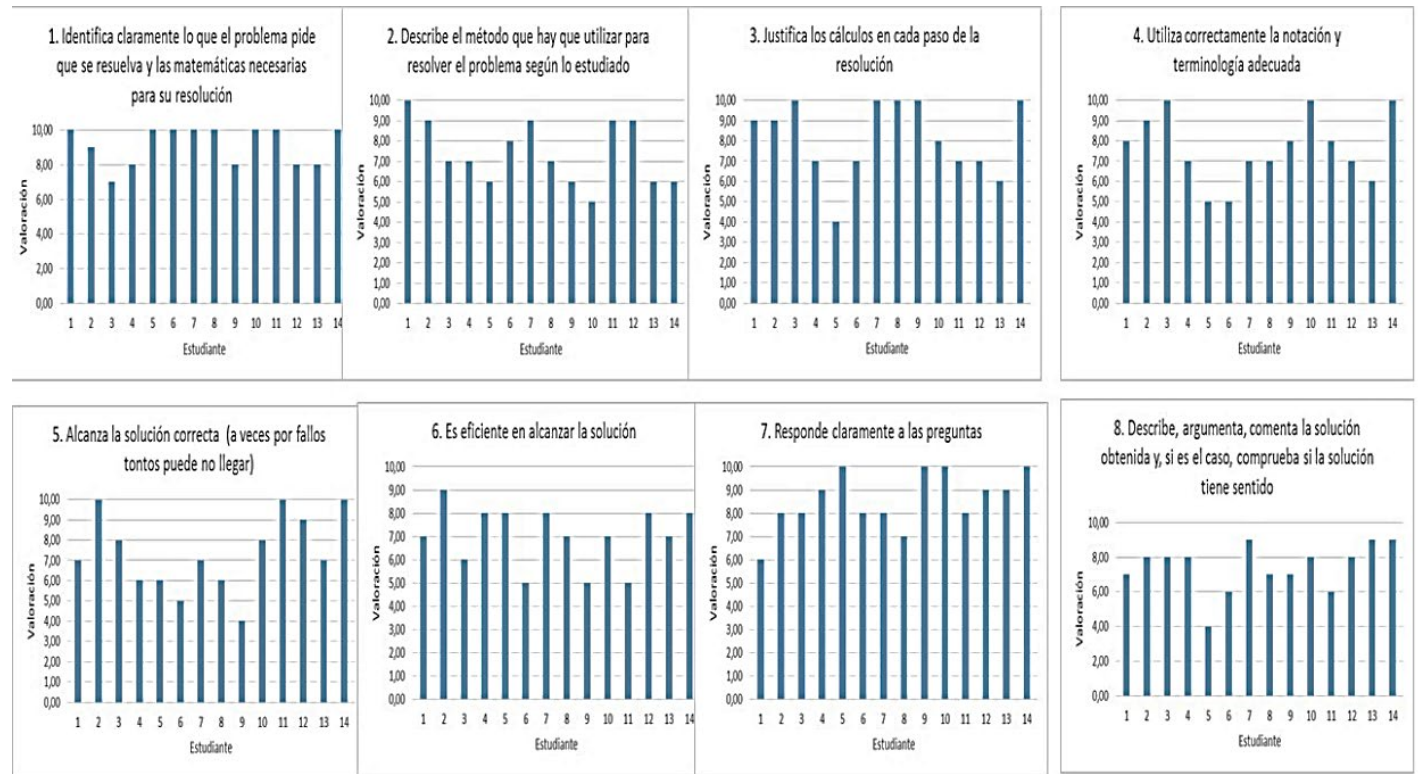

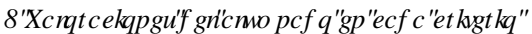

\section{Resultados}

\subsection{Análisis de la valoración por parte del grupo experto}

En la Tabla 2 se muestra el resumen estadístico de las valoraciones obtenidas. Estas valoraciones se entienden como umbrales mínimos requeridos para adquisición de la competencia, y que representaran en secciones posteriores el ideal. Como se observa, los criterios más valorados se corresponden con la parte de conclusión, es decir, los criterios 7 y 8 , en los que el alumnado debe responder claramente a las preguntas planteadas, comentar la solución y comprobar la idoneidad de esta. También destaca el primer criterio en el que se debe identificar lo que el problema solicita y las herramientas necesarias para su resolución. El menos valorado es el criterio 5 , en el sentido de que a veces por fallos tontos se puede no llegar a la solución correcta. La mayor disparidad de opiniones se encuentra en el criterio 6 y 3 , respecto a la forma de alcanzar la solución y la justificación de los pasos en la resolución. Se refleja la normalidad de los datos salvo en el criterio 7 (curtosis estandarizada fuera del rango).

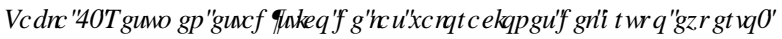

\begin{tabular}{|c|c|c|c|c|c|c|c|c|}
\hline [ & \&UMURा & \&UMURD। & \&UMURT & \&UMURDI & \&UMURा & \&UMURD & \&UMURाए & \&UMURT! \\
\hline 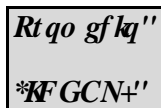 & 8,56 & 7,67 & 7,67 & 8,0 & 7,44 & 7,56 & 9,0 & 8,78 \\
\hline 0 HGDQD & 8,0 & 8,0 & 7,0 & 8,0 & 7,0 & 8,0 & 9,0 & 9,0 \\
\hline $\begin{array}{|ll|}0 & R G D\end{array}$ & 8,0 & $7,0-8,0$ & 7,0 & $7,0-8,0-9,0$ & 7,0 & 8,0 & 9,0 & 9,0 \\
\hline ' HMIDFyQ & 0,726 & 0,707 & 1,22 & 0,866 & 0,882 & 1,33 & 0,5 & 0,667 \\
\hline \begin{tabular}{|l|l|l|l|l|}
0 \\
\end{tabular} & 8,0 & 7,0 & 6,0 & 7,0 & 6,0 & 5,0 & 8,0 & 8,0 \\
\hline 0 i [ IPR R & 10,0 & 9,0 & 10,0 & 9,0 & 9,0 & 9,0 & 10,0 & 10,0 \\
\hline 5 DQJR & 2,0 & 2,0 & 4,0 & 2,0 & 3,0 & 4,0 & 2,0 & 2,0 \\
\hline
\end{tabular}




\subsection{Análisis de la valoración por parte del alumnado}

La Tabla 3 muestra el resumen del análisis estadístico de dichas valoraciones. En general el alumnado valora más el criterio 1 correspondiente a identificar lo que el problema pide y las matemáticas necesarias para su resolución, seguido del criterio 7 sobre el responder claramente a las preguntas planteadas y en tercer lugar el criterio 3 sobre la justificación de los cálculos en cada paso de la resolución. En media, a lo que menos dan relevancia es al criterio 6 , es decir, parece que se le dé menos importancia al método seguido para alcanzar la solución, lo importante es llegar a ella, independientemente de si el método elegido es más costoso y/o menos rápido.

Se aprecia gran variabilidad de opiniones en todos los criterios, en particular en los criterios 5 (alcanzar la solución correcta) y 3 (justificar los cálculos en cada paso).

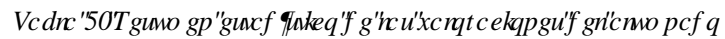

\begin{tabular}{|c|c|c|c|c|c|c|c|c|}
\hline [ & \&UMURT & $\& U M U R \square$ & \&UMURT & \&UसURा & $\& U W U R \square$ & $\& U M A R \square$ & \&UसURा & \&UUART \\
\hline Promedio & 9,14 & 7,43 & 8,14 & 7,64 & 7,36 & 6,92 & 8,57 & 7,43 \\
\hline Mediana & 10,0 & 7,0 & 8,5 & 7,5 & 7,0 & 7,0 & 8,5 & 8,0 \\
\hline Moda & 10,0 & $6,0-9,0$ & 10,0 & 7,0 & 7,0 & 8,0 & 8,0 & 8,0 \\
\hline Desviación & 1,1 & 1,55 & 1,88 & 1,69 & 1,91 & 1,32 & 1,22 & 1,4 \\
\hline Mínimo & 7,0 & 5,0 & 4,0 & 5,0 & 4,0 & 5,0 & 6,0 & 4,0 \\
\hline Máximo & 10,0 & 10,0 & 10,0 & 10,0 & 10,0 & 9,0 & 10,0 & 9,0 \\
\hline Rango & 3,0 & 5,0 & 6,0 & 5,0 & 6,0 & 4,0 & 4,0 & 5,0 \\
\hline
\end{tabular}

\subsection{Comparación entre la valoración del grupo experto y la del alumnado}

Respecto a las diferencias entre las valoraciones en media del grupo experto (el ideal) y del alumnado se tienen los resultados de la Tabla 4 y de la Figura 7.

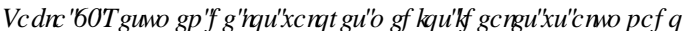

\begin{tabular}{|c|l|l|l|l|l|l|l|l|}
\cline { 2 - 8 } \multicolumn{1}{c|}{} & Criterio 1 & Criterio 2 & Criterio 3 & Criterio 4 & Criterio 5 & Criterio 6 & Criterio 7 & Criterio 8 \\
\hline IDEAL & $\mathbf{8 , 5 6}$ & $\mathbf{7 , 6 7}$ & $\mathbf{7 , 6 7}$ & $\mathbf{8}$ & $\mathbf{7 , 4 4}$ & $\mathbf{7 , 5 6}$ & $\mathbf{9}$ & $\mathbf{8 , 7 8}$ \\
\hline $\begin{array}{l}\text { Promedio } \\
\text { alumnado }\end{array}$ & 9,14 & 7,43 & 8,14 & 7,64 & 7,36 & 6,92 & 8,57 & 7,43 \\
\hline
\end{tabular}

Ordenando los criterios de mayor a menor valoración, se aprecia que en ambos casos se valorar más los criterios 1,7 y 4 (identificar lo que el problema pide, responder claramente a las preguntas planteadas respectivamente y utilizar correctamente la notación y terminología). No se coincide en prioridad con el criterio 8 (describir, argumentar, comentar la solución) al que el profesorado asigna una valoración ideal de 8,78 (segundo lugar), mientras que el alumnado le asigna un 7,43 (quinto lugar). De hecho respecto de las diferencias de valoración, destaca el criterio 8 con una diferencia de 1,35 entre el ideal y la media valorada por el alumnado (8,78 del ideal sobre el 7,43 del alumnado). Se observa también la casi coincidencia en valoración de los criterios 5, 2 y 4 (por orden de más a menos coincidencia). Por tanto se valora de manera similar el que se alcance la solución correcta (7,44 del ideal, frente al 7,36 del alumnado), 
la descripción del método de resolución (7,67 del ideal frente al 7,43 del alumnado) y el uso correcto de la notación y terminología (8 y 7,64).

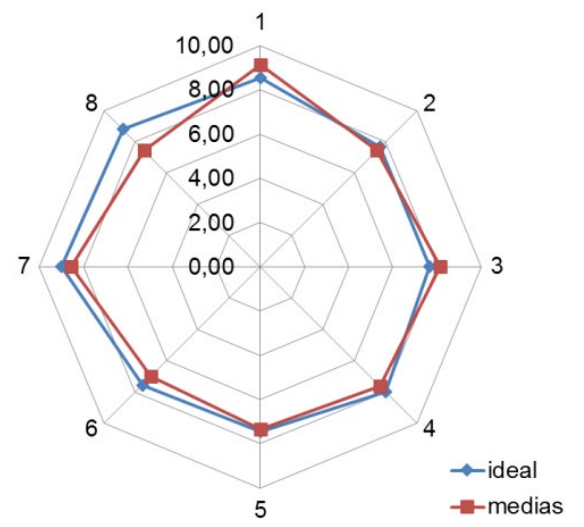

) IJWI\&RP SDWQCRIHOIGHDORQLCVIP HADVGHODOXPQDCR]

Puede apreciarse que el alumnado sobrevalora (en media), respecto del ideal, el criterio $1 \mathrm{y}$ el criterio 3, dándole pues más importancia a la identificación de lo que pide el problema, las herramientas para su resolución y el detallar los pasos en la resolución. De hecho, destaca la sobrevaloración del criterio 1 (identificar lo que el problema pide), en el que 9 de los 14 estudiantes lo han valorado con una nota superior a la del ideal.

Respecto a la infravaloración respecto del ideal, el alumnado, en media, da menos importancia al criterio 8 (describir, argumentar, comentar la solución), con 11 estudiantes que lo infravaloran respecto del ideal, seguido de los criterios 6 (ser eficiente en alcanzar la solución), y 4 (utilizar correctamente la notación y terminología), con 7 estudiantes con valoraciones más bajas que el ideal.

Contrastando si hay diferencias significativas entre las medias de las dos muestras (grupo experto vs alumnado), para un nivel de significación del 95\% en cada criterio, se obtienen los datos de la Tabla 5.

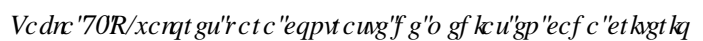

\begin{tabular}{|l|l|l|l|l|l|l|l|l|}
\hline CRITERIO & 1 & 2 & 3 & 4 & 5 & 6 & 7 & 8 \\
\hline P_VALOR & 0,178 & 0,668 & $0,002 *$ & 0,866 & 0,907 & 0,27 & 0,329 & $0,013^{*}$ \\
\hline
\end{tabular}

Por tanto, se puede afirmar que los criterios 3 (justificar los cálculos en cada paso) y 8 (describir, argumentar, comentar la solución) manifiestan valoraciones diferentes entre el grupo experto y el alumnado. Estas diferencias indican la necesidad de estudiar en un futuro a qué puede deberse esta diferencia.

\subsection{Percepción del alumnado vs ideal}

Uno de los objetivos específicos del PIME 19-20/190 era el de realizar encuestas competenciales a nuestro estudiantado y medir la percepción que tiene el alumnado sobre el cumplimiento de la competencia, estudiando las posibles deviaciones respecto de la evaluación del profesor o profesora. En este apartado consideraremos los resultados de la encuesta pasada al alumnado para recoger su percepción respecto a su cumplimiento en cada uno de los criterios consensuados. Invalidamos una de las contestaciones por no calificarlas entre 0 y 10 , sino de forma cualitativa, por tanto en este caso solo se consideran 13 estudiantes. 
La matemática fuzzy o borrosa permite establecer la idoneidad de una persona a un perfil, ya que se exige que el candidato reúna ciertas cualidades que lo hagan adecuado para el puesto adecuado. El procedimiento seguido es primero establecer el perfil idóneo para cada cualidad, segundo establecer el perfil de cada candidato y por último determinar el grado de cercanía entre los perfiles idóneos para cada cualidad y los de cada candidato (Grau, 2006). En nuestro estudio, para analizar el ajuste entre los datos del alumnado y el ideal del profesorado utilizaremos esta idea.

En 1965 Lofti A. Zadeh define el concepto de conjunto fuzzy (borroso o difuso) caracterizándolo por una función de pertenencia que asigna a cada objeto de un conjunto ; no vacío un grado de pertenencia entre 0 y 1. Para este trabajo, fijado $;=\left\{L_{M}: M=1,2, \ldots Q\right\}$, consideramos como conjunto borroso sobre ; पal de la forma $\$=\left\{\left(\left[_{M} I_{\$}\left(\left[_{M}\right)\right): M=1,2, \ldots Q\right\}\right.\right.$, para $I_{\$}\left(\left[_{M}\right) \in[0,1], M 1,2, \ldots Q\right.$ o simplificando la notación, a $\$=\left(I_{\$}\left(\left[_{1}\right), I_{\$}\left(\left[_{2}\right), \ldots, I_{\$}\left(\left[_{Q}\right)\right)\right.\right.\right.$.

En este trabajo consideramos el conjunto de criterios $;=\left\{L_{M}: M=1,2, \ldots 8\right\}$, representando $\left[_{M}\right.$ el criterio $M M 1,2, \ldots \square \square$ Nuestro conjunto fuzzy ideal sobre ; , al que consideramos como perfil idóneo, es , $=\left(I,\left(\left[_{1}\right), I,\left(\left[_{2}\right), \ldots I,\left(\left[_{8}\right)\right)\right.\right.\right.$ en el que $I,\left(\left[_{M}\right)\right.$ se corresponde con la nota ideal del criterio Mdividida por 10 , y que en nuestro caso es

$$
,=\{0.856,0.767,0.767,0.8,0.744,0.756,0.9,0.878\}
$$

Consideremos otro conjunto fuzzy $\$=\left(I_{\$}\left(\left[_{1}\right), I_{\$}\left(\left[_{2}\right), \ldots, I_{\$}\left(\left[_{Q}\right)\right)\right.\right.\right.$ sobre el conjunto de criterios; , que se corresponde a las valoraciones de un o una estudiante, que sin pérdida de generalidad suponemos de 0 a 1. Para determinar el grado de cercanía del perfil idóneo o ideal al de cada estudiante, pueden considerarse varios métodos, que en general establecen una ordenación de candidatos ante un determinado perfil empresarial, deportivo,... (Grau, 2006). En particular Canós-Daros et al. (2021) utilizan un índice de adecuación que ordena al alumnado según estén más o menos cerca del ideal de alcance de la CT Gestión y planificación del tiempo, penalizando al alumnado que no alcanza el nivel ideal y premiando de forma distinta al que sobrepasa el nivel ideal en cada criterio. En nuestro caso, al suponer que los niveles ideales son mínimos requeridos, todo aquel que en un criterio alcance el mínimo exigido se considerará con el mismo premio que el que lo sobrepasa. En este caso no se pretende establecer una ordenación para elegir el candidato más cercano al ideal, sino tener un valor entre 0 y 1 que nos indique el grado de adquisición de la CT en cada estudiante. Para analizar el ajuste entre los datos del alumnado y el ideal del profesorado utilizaremos uno de los conceptos de la matemática fuzzy conocido como índice de adecuación de $\$$ en , (Gil, 1996), dado por la expresión

$$
\& D(\$)=\frac{\sum_{M \neq 1}^{8} \cdot{ }_{M}}{8}
$$

siendo $~_{M}=\operatorname{PtQPP} R\left(1,\left(1+I_{\$}\left(\left[_{M}\right)-I_{,}\left(\left[_{M}\right)\right)\right)\right.\right.$ para $M 1,2, \ldots$ पा o equivalentemente:

- $\quad{ }_{M} 1$ si $I,\left(\left[_{M}\right)-I_{\$}\left(\left[_{M}\right) \leq 0 \square\right.\right.$

- . ${ }_{M} 1-\left(I,\left(\left[_{M}\right)-I_{\$}\left(\left[_{M}\right)\right)=1+I_{\$}\left(\left[_{M}\right)-I_{,}\left(\left[_{M}\right)\right.\right.\right.\right.$, si $I,\left(\left[_{M}\right)-I_{\$}\left(\left[_{M}\right)>0 \square\right.\right.$ 
Si un o una estudiante llega o sobrepasa el nivel ideal en un criterio $\left[_{M}\right.$, no sufre penalización, siendo . ${ }_{M} 1$. Pero si no alcanza el ideal es penalizado, siendo en este caso ${ }_{M} 1-\left(I,\left({ }_{M}\right)-I_{\$}\left(\left[_{M}\right)\right)\right.$, es decir, se le penaliza restando al 1 lo que dista del ideal. Para aclararlo y ver su funcionamiento, consideremos el ejemplo de la Tabla 6, en la que aparecen los niveles ideales junto con los resultados de la percepción de un o una estudiante respecto a su cumplimiento en cada uno de los criterios exigidos y en la Figura 8 se observa gráficamente esta comparación.

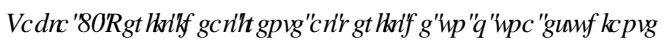

\begin{tabular}{|c|c|c|c|c|c|c|c|c|}
\hline & $L_{1}$ & $L_{2}$ & $L_{3}$ & $L_{4}$ & $L_{5}$ & $L_{6}$ & $L_{7}$ & $L_{8}$ \\
\hline IDEAL $I$, & 0.856 & 0.767 & 0.767 & 0.8 & 0.744 & 0.756 & 0.9 & 0.878 \\
\hline Estudiante $I_{\$}$ & 0.8 & 0.7 & 0.8 & 0.5 & 0.9 & 0.6 & 0.8 & 0.8 \\
\hline
\end{tabular}

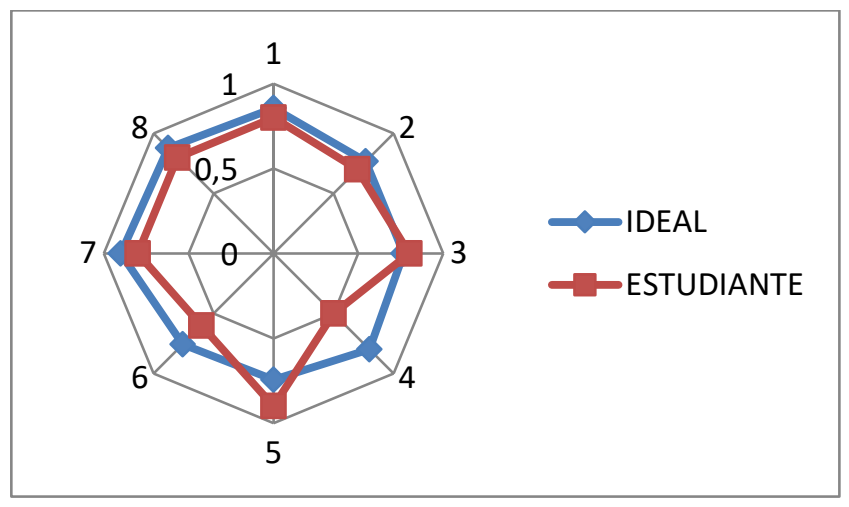

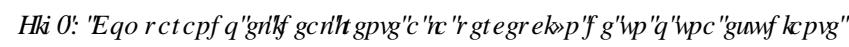

El cálculo de . $M M 1,2, \ldots 8$ para cada estudiante y del coeficiente de adecuación puede verse en la Tabla 7.

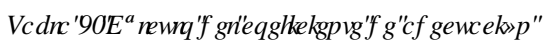

\begin{tabular}{|c|c|c|c|c|c|c|c|c|c|}
\hline $\bar{M}$ & 1 & 2 & 3 & 4 & 5 & 6 & 7 & 8 & \\
\hline$I,\left(\left[_{M}\right)-I_{\$}\left(\left[_{M}\right)\right.\right.$ & 0.06 & 0.07 & -0.03 & 0.3 & -0.16 & 0.16 & 0.1 & 0.08 & \\
\hline . $\mathrm{j} \square$ & 0.94 & 0,93 & 1 & 0.7 & 1 & 0.84 & 0.9 & 0.92 & $\& D=0.9$ \\
\hline
\end{tabular}

Por tanto, para el o la estudiante, el grado de adquisición de la competencia sería de $\& D=\mathbf{0 . 9}$. Cabe señalar que $\& D$ es mayor o igual al promedio de los valores $I_{\$}\left(L_{M}\right)$ de cada estudiante (sin tener en cuenta el ideal), siendo igual cuando todos los ideales son 1. De hecho puede probarse que:

$$
\& D \geq \operatorname{promedio}+\frac{\sum_{M I_{s}<I,}\left(1-I_{,}\left(\left[_{M}\right)\right)\right.}{Q}, \text { para Qcriterios (2) }
$$

Así pues el coeficiente de adecuación de $\$$ en , $\& D$, representa una generalización de la media aritmética de notas, tan utilizada en docencia. Para el o la estudiante de la Tabla 6 se tiene $\& D=0.9$, mientras que su 
nota promedio sería menor, de 0,74 , es decir, de 0,74 considerando como ideal el 1 en todos los criterios (o 10). El resultado del grado de percepción de los 13 estudiantes se refleja en la Tabla 8 .

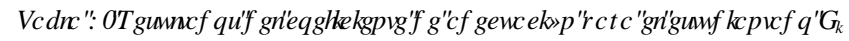

\begin{tabular}{|c|c|c|c|c|c|c|c|c|c|c|c|c|c|}
\hline & E1 & E2 & E3 & E4 & E5 & E6 & E7 & E8 & E9 & E10 & E11 & E12 & E13 \\
\hline Ca & 0,8 & 0,98 & 0,92 & 1 & 0,97 & 0,79 & 0,91 & 0,88 & 0,98 & 0,8 & 0,86 & 0,93 & 0,88 \\
\hline
\end{tabular}

Este coeficiente de adecuación puede servir para calificar la adquisición de la correspondiente competencia del siguiente modo: D si $\& D<0.25$; C si $\& D \in[0.25,0.5[$; B si $\& D \in[0.5,0.75[$ y A si $\& D>0.75$. Siguiendo este método, la percepción de la adquisición de la CT-03 en todo el alumnado es haber alcanzado la calificación A.

Respecto a la diferencia de resultados en cuanto a la calificación de la competencia utilizando el coeficiente de adecuación $\& D$ o el promedio, tal y como se ha indicado se sabe que Promedio $\leq \square \& D \|$ Este hecho puede repercutir en la calificación cualitativa final de la competencia. Como ejemplo mostramos en la Tabla 9 los resultados de una simulación realizada con 30 estudiantes cuyas valoraciones se han obtenido de forma aleatoria. Considerando el ideal propuesto en este trabajo, la calificación de la competencia solo coincide en un $36,7 \%$ de la muestra.

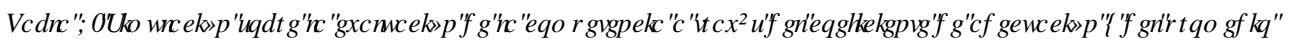

\begin{tabular}{|l|l|l|l|l|l|l|l|l|l|l|l|l|l|l|l|l|l|l|l|l|l|l|l|l|l|l|l|l|l|l|l|}
\hline Ca & A & B & A & B & C & A & A & A & A & B & B & C & A & B & B & A & C & B & B & B & B & B & C & C & B & B & B & B & B & B \\
\hline Media & B & B & B & C & D & B & B & B & B & B & C & C & B & C & C & B & C & B & C & B & C & B & C & C & C & C & C & B & C & B \\
\hline
\end{tabular}

Ampliamos la simulación al caso de una clase de 100 estudiantes. Para tener una idea del comportamiento que puede tener la variable $\mathrm{IG}=$ número de estudiantes de la clase que obtienen la misma valoración cualitativa de la CT con el coeficiente de adecuación y con el promedio, realizamos 500 repeticiones similares a la del ejemplo descrito, obteniendo 500 valores de IG. En el análisis de dichos valores se obtiene una media muestral de 33.22 (aproximadamente solo en una tercera parte coinciden las valoraciones) y desviación de 4.79. En el análisis de esta muestra, tanto el valor del sesgo estandarizado como el de la curtosis se encuentran dentro del rango esperado para datos provenientes una distribución normal. En la Figura 9 observamos el histograma.

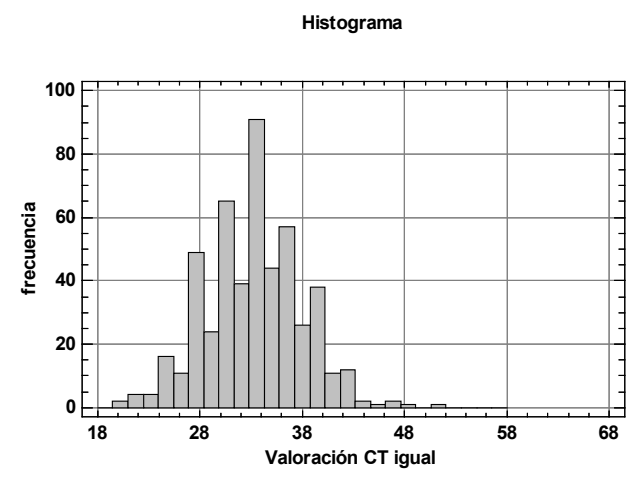

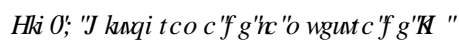




\section{Conclusiones}

En este trabajo se ha realizado una valoración de cada uno de los indicadores o criterios establecidos para la evaluación de la CT-03 Análisis y resolución de problemas por el grupo de profesores del PIME 1920/190, a través de un grupo experto, estableciendo un ideal o de mínimos umbrales, y a través del alumnado de una asignatura de matemáticas del GTI. En la comparación de estas valoraciones se observan diferencias de valoración entre el criterio 8 (describir, argumentar, comentar la solución obtenida) con un 8,78 del ideal sobre el 7,43 del alumnado. Se observa también la casi coincidencia en valoración de los criterios 5, 2 y 4, valorándose por tanto de manera similar el que se alcance la solución correcta, la descripción del método de resolución y el uso correcto de la notación y terminología. Por otro lado se constata la existencia de diferencias significativas entre las medias de las valoraciones del grupo experto y alumnado en el criterio 3 (justificar los cálculos en cada paso) y 8 (describir, argumentar, comentar la solución), diferencias que indican la necesidad de estudiar en un futuro a qué puede deberse.

Respecto a la percepción del alumnado en cuanto al cumplimiento de los indicadores establecidos para la evaluación de la CT-03 Análisis y resolución de problemas, se ha calculado un coeficiente de adecuación que ha permitido conocer el ajuste entre el alumnado y el ideal de adquisición de la competencia dado por el grupo experto.

Este trabajo da lugar a poder utilizar los indicadores estudiados a través de una rúbrica para poderla implementar en la evaluación de la adquisición de la CT-03 con la utilización del coeficiente fuzzy de adecuación al ideal. En caso de no partir de un ideal de mínimos umbrales, este coeficiente se reduce a la media aritmética o promedio, aunque hay que tener en cuenta las diferencias que pueden producirse en la valoración cualitativa final de la CT. Es cierto que esta tarea puede resultar laboriosa si el grupo de estudiantes es numeroso y/o si es elevado el número de actividades formativas propuestas para la evaluación de la CT. En Pérez-Sánchez et al., (2017) se utiliza la evaluación por pares, con una plantilla que debe rellenar cada estudiante corrector como guía de apoyo, idea que puede ser de utilidad para actividades formativas propuestas como Tareas. Habrá que buscar por tanto un equilibrio para evaluar sin morir en el intento. Y a partir de ahí, en un futuro, también es interesante poder comparar la evaluación final de la CT03 con la propia percepción del alumnado y estudiar si es el caso las posibles deviaciones.

\section{Agradecimientos}

Los autores quieren agradecer la ayuda económica y el apoyo institucional recibidos de la Universitat Politècnica de València a través del proyecto PIME 19-20/190.

\section{Referencias}

ALVAREZ BLANCO, S.; BOSCH ROIG, I.; JORDAN LLUCH, C.; LLORET MAURI, J.; MENDOZA ROCA, JA.; ROMERO PÉREZ, L.; SANABRIA CODESAL, E. (2016). "Análisis y comparación de la Competencia Transversal

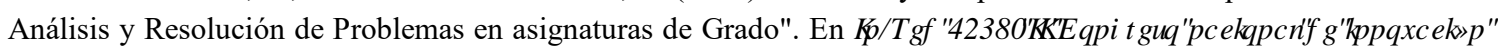
HEXFDUSD ID GRFHPFD HQ UHE Valencia. Editorial Universitat Politècnica de València. https://doi.org/10.4995/INRED2016.2016.4348

BOIGUES PLANES, F.J., ESTRUCH FUSTER, V., VIDAL MELO, A. (2019). "La comunicación efectiva en matemáticas: una manera de educar en competencias en la universidad" en Roig-Vila, R. 5HGHWGH,QYHMWDFIyQH ,QQRYDFIyQHQ' RFHQFD8 QIYHUWDUD. Volumen 2019. Alicante: Universidad de Alicante, Instituto de Ciencias de la Educación (ICE), 2019. ISBN 978-84-09-07186-9, pp. 7-20

CALAFAT MARZAL, MC.; CABEDO MALLOL, V.; CASTELlANO I CERVERA, V.; COMPANY CARRETERO, FJ.; MARÍN SÁNCHEZ, MDM.; PÉREZ ALONSO, Y. (2019). "Formación en competencias trasversales en el Grado de Gestión y Administración Pública: Análisis y resolución de problemas". En -,' ' 2 凹, ( 


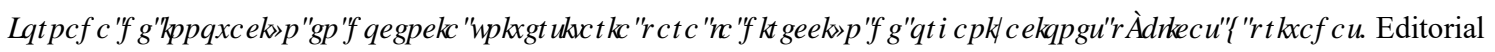
Universitat Politècnica de València. 135-144. https://doi.org/10.4995/JIDDO2019.2019.10181

CANOS-DAROS, L., SANTANDREU-MASCARELL, C. Y VIDAL-MELO, A. (2021). "La medida de la competencia transversal. Planificación y gestión del tiempo en el grado en Ingeniería de Telecomunicaciones, Sonido

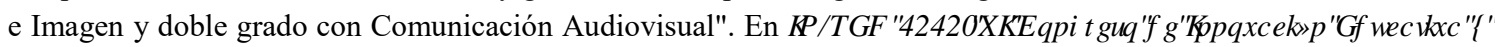
' RFHQWD HQ 5HG Valencia. Editorial Universitat Politècnica de València. 972-983. https://doi.org/10.4995/INRED2020.2020.11955

CERVELLÓ ROYO, RE.; MARQUÉS PÉREZ, I. (2016). "Mejora del sistema de evaluación de las prácticas con ordenador en la asignatura de Matemáticas Financieras I en el Grado de ADE incorporando las competencias

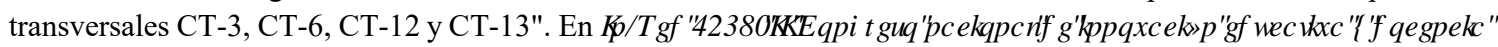
HQUHG Editorial Universitat Politècnica de València. https://doi.org/10.4995/INRED2016.2016.4419

CORTES LOPEZ, JC.; MATEOS-RONCO, A.; SANTAMARIA NAVARRO, C.; VILLANUEVA MICO, RJ. (2019). Evaluación de la competencia transversal "Análisis y resolución de problemas (CT3) en la titulación de GADE de la Facultad de ADE de la Universitat Politècnica de Valéncia". ( Q-,', ' 2 प, -RLQDDD GH IQQRYDFIYQHQ GRFQFLD

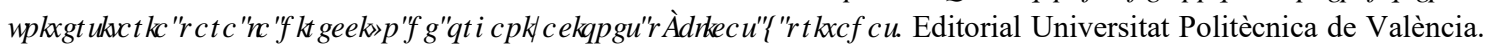
24-31. https://doi.org/10.4995/JIDDO2019.2019.10184

GRAU GRAU, ALFREDO J. (2006). "Aplicación de la matemática borrosa a la gestión deportiva" en 680 \$प5 HIMD

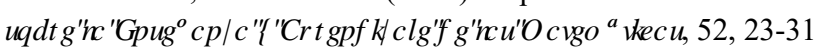

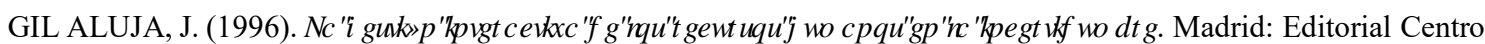
de Estudios Ramón Areces.

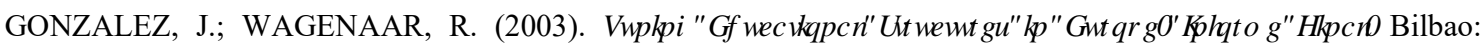
Universidad de Deusto. http://www.ub.edu/cubac/sites/default/files/tuning_educational_structures_espanyol_0.pdf. [Consulta: 19 de febrero de 2021].

PEREZ BERNABEU, E.; SELLES CANTO, M.Á.; GISBERT SOLER, V.; SANCHEZ CABALLERO, S. (2016). "Un

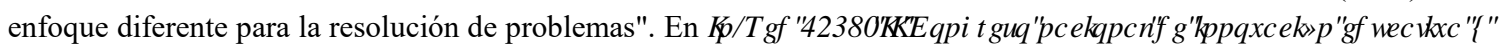
GRFPFWHQUHG Editorial Universitat Politècnica de València. https://doi.org/10.4995/INRED2016.2016.4309

PEREZ-SANCHEZ, M.; PEREZ-SANCHEZ, M.; LOPEZ JIMENEZ, PA. (2017). ¿Puede un conjunto de tareas

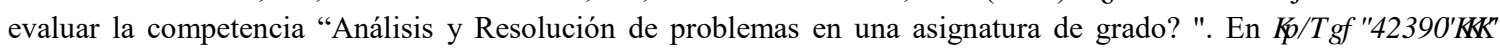
\&RQJ UHR 1 DFIRQDOCHIQQRVDFIY QHGXFDUMDI ICHCRFHQFIDHQUHG Editorial Universitat Politècnica de València. 13221334. https://doi.org/10.4995/INRED2017.2017.6869

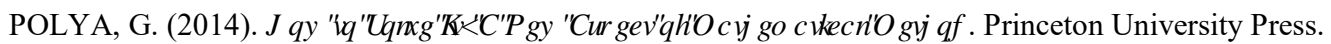

SKEMP, R. R. (1978) "Relational understanding and instrumental understanding" en \$UWKP HMFUADFHU 26, 3, p. 915 .

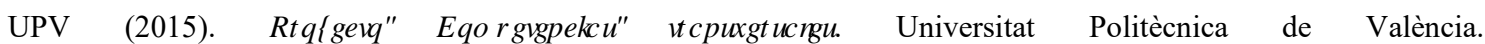
https://www.upv.es/entidades/ICE/info/Proyecto_Institucional_CT.pdf [Consulta: 19 de febrero de 2021]

(c)) BY-NC-ND 2021, Universitat Politècnica de València

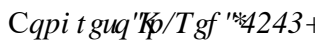

